Critical inclination for $\mathrm{A} / \mathrm{C}$ transition in inverted falling films Physics of Fluids

\title{
Critical inclination for Absolute/Convective instability transition in inverted
}

\section{falling films}

Benoit Scheid, ${ }^{1, \text { a) }}$ Nicolas Kofman, ${ }^{2, \text { b) }}$ and Wilko Rohlfs ${ }^{3,4, c)}$

1) TIPs, Université Libre de Bruxelles, C.P. 165/67,

Avenue F. D. Roosevelt 50, 1050 Bruxelles, Belgium

2) Laboratory of Fluid Mechanics and Instabilities, Station 9, EPFL, 1015 Lausanne, Switzerland

${ }^{3)}$ Institute of Heat and Mass Transfer, RWTH Aachen University, Augustinerbach 6, 52056 Aachen, Germany

4) Department of Mechanical Engineering, Massachusetts Institute

of Technology, 77 Massachusetts Avenue, Cambridge MA, $U S$

(Dated: 7 janvier 2016) 
Critical inclination for $\mathrm{A} / \mathrm{C}$ transition in inverted falling films

Liquid films flowing down the underside of inclined plates are subject to the interaction between the hydrodynamic and the Rayleigh-Taylor (R-T) instabilities causing a patterned and wavy topology at the free surface. The R-T instability results from the denser liquid film being located above a less dense ambient gas, and deforming into an array of droplets, which eventually drip if no saturation mechanism arises. Such saturation mechanism can actually be provided by a fluid motion along the inclined plate. Using a weighted integral boundary layer model, this study examines the critical inclination angle, measured from the vertical, that separates regimes of absolute and convective instability. If the instability is of absolute type, growing perturbations stay localized in space potentially leading to dripping. If the instability is of convective type, growing perturbations move downwards the inclined plate, forming waves and eventually, but not necessarily, droplets. Remarkably, there is a minimum value of the critical angle below which a regime of absolute instability cannot exist. This minimum angle decreases with viscosity : it is about $85^{\circ}$ for water, about $70^{\circ}$ for silicon oil, and reaches a limiting value for liquid with a viscosity larger than about 1000 times the one of water. It results that for any fluid, absolute dripping can only exist for inclination angle (taken from the vertical) larger than $57.4^{\circ}$.

Keywords: Falling film, Rayleigh-Taylor, Absolute and Convective Instability, Dripping

\footnotetext{
a)Electronic mail: bscheid@ulb.ac.be

b)Electronic mail: nicolas.kofman@epfl.ch

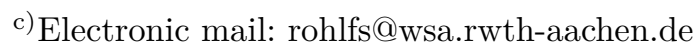


Critical inclination for $\mathrm{A} / \mathrm{C}$ transition in inverted falling films

\section{INTRODUCTION}

Falling liquid films on vertical plates or on the upper side of inclined plates are present in many technical applications involving heat and mass transfer, and as such they are well studied ${ }^{1-3}$. Owing to an inherent unstable flow above a critical inclination angle, falling films are characterized by a wavy and distorted topology exhibiting different types of vortices in the trough ${ }^{4}$ and the crest $^{5}$ of the wave. These instabilities are always of convective type ${ }^{3}$, such that surface perturbations grow in space (in flow direction) and not locally in time, which is confirmed by experiments ${ }^{6}$. For the inverse case of a film flowing down the bottom side of an inclined plate, the flow should also be of convective type if the inclination angle is close to the vertical. However, in the limiting case of a horizontal plate, the Rayleigh-Taylor ${ }^{7}$ (R-T) instability deforms the surface. The entire system is described by a balance between destabilizing gravitational forces, and stabilizing surface tension forces. The flow is thus of absolute type if the inclination angle is sufficiently close to the horizontal, meaning that perturbations grow locally and are not convected by the flow ${ }^{8}$. As a consequence, there should exist a critical (fluid and flow rate specific) angle at which the instability changes from absolute to convective type. This is what has recently been demonstrated by Brun et al. ${ }^{9}$ in the limit of negligible inertia and viscous extensional stress strictly valid for low Reynolds number flows. Extending their study to large Reynolds number flows is the aim of this work, which will show non-monotonic absolute/convective transition contrarily to the previous results.

It is well known that the R-T instability in a static and horizontal set-up does not exhibit a saturation mechanism ${ }^{10}$, such that either droplet detachment or film rupture occur within a finite time period ${ }^{11}$. Saturation of the R-T instability and consequently a suppression of dripping can be achieved in different ways, such as applying oscillations in vertical ${ }^{12}$ or horizontal ${ }^{13}$ direction, an electric field, or tem- 
Critical inclination for $\mathrm{A} / \mathrm{C}$ transition in inverted falling films

perature gradients ${ }^{14-16}$. In close relation to falling films, Babchin et al. ${ }^{17}$ have demonstrated that an convective flow in horizontal direction (due to a moving plate at a constant velocity) can result in saturation. This saturation is a result of a non-linear flow-induced and surface-tension-assisted mechanism.

The formation of the three-dimensional R-T instability in an inverted film flow was studied by Lin et al. ${ }^{18}$ using a model based on lubrication approximation with neglected effects of inertia. Contrarily to the film flow considered in this study, Lin et $a l$. have a non-wetted plate as an initial condition. Although the main focus of their study is on the development of finger-like patterns, they also consider a film destabilized by a Rayleigh-Taylor type instability. They identify for low values of the dimensionless inclination number a propagation front which breaks up into fingers. A further characteristic of the flow are large droplet-like structures which move downwards the plate with a wave speed much faster than the propagation speed of the fingers.

On conditions that the intensity of the saturation mechanism is not sufficiently strong, and e.g. in the case of an imposed flow rate, the boundary between the convective and absolute instability will separate the regime of immediate dripping (see Fig. 1c), occurring already in the vicinity of the inlet, from the regime where dripping eventually, but not necessarily, occurs after a sufficiently long inlet length (see Fig. 1b). In a similar way the absolute/convective (A/C) boundary separates the regimes of droplet formation in a viscous liquid jet ${ }^{19,20}$, e.g. droplet formation at the nozzle exit or downstream jet breakup, both owing to Plateau-Rayleigh instability.

In Section II, a set of low-dimensionality models based on the weighted integral boundary layer approach will be proposed and hierarchized in terms of their degree of approximation. In Section III, we will present the methodology to identify the $\mathrm{A} / \mathrm{C}$ transition of a falling liquid film on the underside of an inclined plate subject to R-T instability. A special characteristic of this flow configuration is that both the 
Critical inclination for $\mathrm{A} / \mathrm{C}$ transition in inverted falling films

R-T instability and the convective liquid transport along the inclined plate are driven by gravity. Thus, the cotangent of the inclination angle taken from the horizontal determines the ratio between the two coupled mechanisms. In Section IV, and after having shown the differences and specificities of the various models, the influence of two different conservation conditions, e.g. imposed film thickness and imposed flow rate on the $\mathrm{A} / \mathrm{C}$ transition is examined. Further, a minimum critical inclination angle for convective instability in falling films is presented. In Section Discussions, we discuss the relevancy of the $\mathrm{A} / \mathrm{C}$ transition in light of time-dependent simulations of wavepackets, whereas conclusions are given in Section VI.

\section{MODELING}

The geometry of an inverted falling film under the field of gravity $g$ is sketched in Fig. 1(a) where $\beta$ is the inclination angle from the horizontal and $\alpha=\beta-\pi / 2$ is the inclination angle taken from the vertical, the two angles being used in the paper. The unperturbed flat film thickness is denoted $h_{N}$, and $q_{N}$ is the specific volumetric flow rate (flow rate per unit width) at the inlet. For the evolution of the film flow, the full second-order model obtained by Ruyer-Quil and Manneville ${ }^{21}$ is considered, whose derivation is briefly detailed in appendix A. This model consists in four coupled evolution equations for the local film thickness $h(x, t)$, the local flow rate $q(x, t)$, and two corrections of the flow rate $s_{1}(x, t)$ and $s_{2}(x, t)$ accounting for the departure from the parabolic velocity profile due to second-order inertia effects. The ordering refers to the classical gradient expansion ${ }^{3}$, such as second-order terms contain second-order derivatives in space $(x)$. Using $h_{N}$ as the scale for the thickness, and $3 q_{N}$ as the scale for the flow rate, the dimensionless equations are

$$
\partial_{t} h=-\partial_{x} q
$$


Critical inclination for $\mathrm{A} / \mathrm{C}$ transition in inverted falling films

(a) Geometry

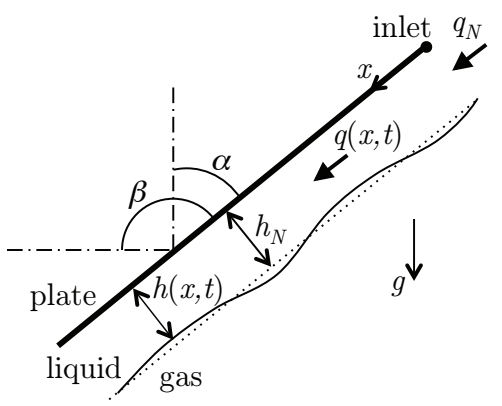

(b) Convective instability

(c) Absolute instability

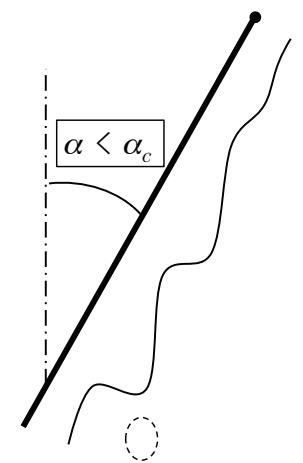

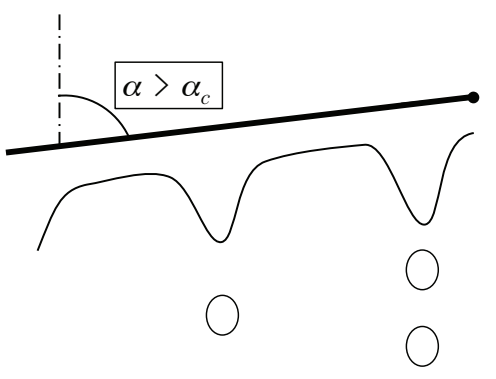

Figure 1: Sketch of an inverted falling film, a situation that is always unstable : (a) any perturbation around the unperturbed flat film solution will grow in time and either be convected with the flow (b) or be localized in space (c), in the case of which dripping already occurs in the vicinity of the inlet in absence of nonlinear saturation. Note that dripping can eventually, but not necessarily, occur in convective instability as represented by the dashed droplet. The critical angle $\alpha_{c}$, separates the two regimes of instability.

$$
\begin{aligned}
\delta \partial_{t} q= & \frac{5}{6} h-\frac{5}{2} \frac{q}{h^{2}}-\frac{5}{6} \zeta h \partial_{x} h+\frac{5}{6} h \partial_{x x x} h+\delta \partial_{t} s_{1}+\delta \partial_{t} s_{2} \\
+ & \delta\left[-4 \frac{q s_{1} \partial_{x} h}{h^{2}}-\frac{18}{7} \frac{q s_{2} \partial_{x} h}{h^{2}}+\frac{13}{3} \frac{s_{1} \partial_{x} q}{h}+\frac{17}{7} \frac{s_{2} \partial_{x} q}{h}\right. \\
& \left.+3 \frac{q \partial_{x} s_{1}}{h}+\frac{27}{14} \frac{q \partial_{x} s_{2}}{h}+\frac{9}{7} \frac{q^{2} \partial_{x} h}{h^{2}}-\frac{17}{7} \frac{q \partial_{x} q}{h}\right] \\
+ & +\left[4 \frac{q}{h^{2}}\left(\partial_{x} h\right)^{2}-\frac{9}{2 h} \partial_{x} q \partial_{x} h-6 \frac{q}{h} \partial_{x x} h+\frac{9}{2} \partial_{x x} q\right], \\
\delta \partial_{t} s_{1}= & \frac{1}{10} h-\frac{3}{10} \frac{q}{h^{2}}-\frac{126}{5} \frac{s_{1}}{h^{2}}-\frac{126}{5} \frac{s_{2}}{h^{2}}-\frac{1}{10} \zeta h \partial_{x} h+\frac{1}{10} h \partial_{x x x} h \\
+ & {\left[-\frac{3}{35} \frac{q^{2} \partial_{x} h}{h^{2}}+\frac{1}{35} \frac{q \partial_{x} q}{h}+\frac{108}{55} \frac{q s_{1} \partial_{x} h}{h^{2}}-\frac{5022}{5005} \frac{q s_{2} \partial_{x} h}{h^{2}}\right.} \\
& \left.-\frac{103}{55} \frac{s_{1} \partial_{x} q}{h}+\frac{9657}{5005} \frac{s_{2} \partial_{x} q}{h}-\frac{39}{55} \frac{q \partial_{x} s_{1}}{h}+\frac{10557}{10010} \frac{q \partial_{x} s_{2}}{h}\right]
\end{aligned}
$$


Critical inclination for $\mathrm{A} / \mathrm{C}$ transition in inverted falling films

$$
\begin{aligned}
&+\eta\left[\frac{93}{40} \frac{q\left(\partial_{x} h\right)^{2}}{h^{2}}-\frac{69}{40} \frac{\partial_{x} h \partial_{x} q}{h}+\frac{21}{80} \frac{q \partial_{x x} h}{h}-\frac{9}{40} \partial_{x x} q\right] \\
& \delta \partial_{t} s_{2}= \frac{13}{420} h-\frac{13}{140} \frac{q}{h^{2}}-\frac{39}{5} \frac{s_{1}}{h^{2}}-\frac{11817}{140} \frac{s_{2}}{h^{2}}-\frac{13}{420} \zeta h \partial_{x} h+\frac{13}{420} h \partial_{x x x} h \\
&+\delta\left[-\frac{4}{11} \frac{q s_{1} \partial_{x} h}{h^{2}}+\frac{18}{11} \frac{q s_{2} \partial_{x} h}{h^{2}}-\frac{2}{33} \frac{s_{1} \partial_{x} q}{h}-\frac{19}{11} \frac{s_{2} \partial_{x} q}{h}+\frac{6}{55} \frac{q \partial_{x} s_{1}}{h}-\frac{288}{385} \frac{q \partial_{x} s_{2}}{h}\right] \\
&+\eta\left[-\frac{3211}{4480} \frac{q\left(\partial_{x} h\right)^{2}}{h^{2}}+\frac{2613}{4480} \frac{\partial_{x} h \partial_{x} q}{h}-\frac{2847}{8960} \frac{q \partial_{x x} h}{h}+\frac{559}{2240} \partial_{x x} q\right],
\end{aligned}
$$

where $\delta$ is the reduced Reynolds number, $\zeta$ is the reduced inclination number and $\eta$ is the viscous extensional number, whose definitions are given in the next section (see Eq. 9). The parameter $\delta>0$ scales for the inertia effect, the parameter $\zeta<0$ for the R-T instability mechanism and the parameter $\eta>0$ for the viscous dissipation in the streamwise direction. As proposed by Ruyer-Quil and Manneville ${ }^{21}$, an adiabatic elimination of the fields $s_{1}=s_{2}=0$ in (1) leads to the following simplified secondorder model :

$$
\begin{aligned}
\partial_{t} h= & -\partial_{x} q \\
\delta \partial_{t} q= & \frac{5}{6} h-\frac{5}{2} \frac{q}{h^{2}}-\delta \frac{17}{7} \frac{q}{h} \partial_{x} q+\left(\delta \frac{9}{7} \frac{q^{2}}{h^{2}}-\frac{5}{6} \zeta h\right) \partial_{x} h+\frac{5}{6} h \partial_{x x x} h \\
& +\eta\left[4 \frac{q}{h^{2}}\left(\partial_{x} h\right)^{2}-\frac{9}{2 h} \partial_{x} q \partial_{x} h-6 \frac{q}{h} \partial_{x x} h+\frac{9}{2} \partial_{x x} q\right] .
\end{aligned}
$$

It is worth mentioning that the terms in square brackets result from the so-called extensional viscous stress, well known in models for thin viscous sheets ${ }^{22}$ for which the extensional term is $4 \partial_{x}\left(h \partial_{x}(q / h)\right)$, where 4 is the so-called Trouton ratio. The only differences here stand in the coefficients of each term (apart for the first one), which account for the integration of a parabolic velocity profile, instead of a uniform velocity profile in the case of a free-standing film. If these second-order viscous extensional terms are further neglected, i.e. taking $\eta=0,(2)$ reduces to the first-order model, which therefore corresponds to the two first line of (2). Finally, neglecting also inertia effects, i.e. for $\delta=0$, the first-order model reduces, by eliminating $q$, to a single long- 
Critical inclination for $\mathrm{A} / \mathrm{C}$ transition in inverted falling films

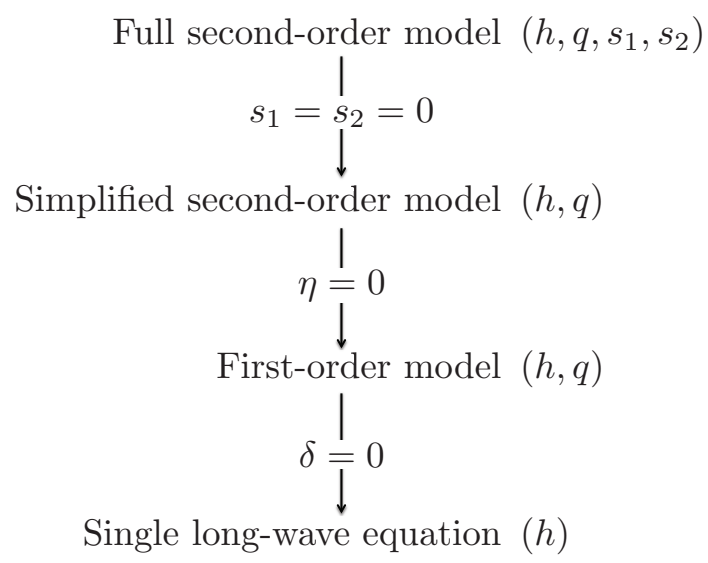

FiguRE 2: Hierarchy of averaged models with corresponding dependent variables in parentheses. Each arrow indicates the step to reduce one model to another. The steps are additive.

wave equation for the film thickness :

$$
\partial_{t} h=-\partial_{x}\left[\frac{h^{3}}{3}\left(1+\partial_{x x x} h-\zeta \partial_{x} h\right)\right] .
$$

This equation is identical to the one considered by Duprat et al. ${ }^{23}$ (except that their $\beta$ parameter equals $-\zeta$ here), even if another physics is involved since the authors considered a film flowing along a fibre, where the absolute instability mode arises due to the Rayleigh-Plateau instability. Equation (3) has also been recently analysed by Brun et al. ${ }^{9}$, who have used another scaling that will be explicated below in Section IV B.

The hierarchy of models that have been introduced above is summarised in Figure 2. Most of the results below are obtained with the full second-order model since it has been shown by Ruyer-Quil and Manneville ${ }^{24}$ to contain all features needed to fit exact linear properties obtained with the Orr-Sommerfeld equations - i.e. the perturbation equations directly obtained from the Navier-Stokes equations. However, the differences between the full second-order model and the three other ones will 
Critical inclination for $\mathrm{A} / \mathrm{C}$ transition in inverted falling films

be first examined in the Section IV, putting in evidence some important influences of the various effects considered, such as inertia at first- and second-order, and viscous extensional stress.

\section{METHODOLOGY}

With the aim to identify the linear absolute/convective $(\mathrm{A} / \mathrm{C})$ transition, the following normal mode perturbations of the dimensionless flat film solution are considered :

$$
\begin{aligned}
& h=1+a e^{i(k x-\omega t),} \\
& q=\frac{1}{3}+b e^{i(k x-\omega t)},
\end{aligned}
$$

where $k=k_{r}+i k_{i}$ and $\omega=\omega_{r}+i \omega_{i}$ are complex wavenumber and complex pulsation, respectively, and $a$ and $b$ are complex amplitudes. For the sake of simplicity, the procedure is detailed below with the simplified second-order model and then extended

to the other models. Inserting (4) into (2), and linearising for $a, b \ll 1$, leads to the following dispersion relation,

$$
k^{2}\left(-\frac{\delta}{7}-\frac{9 i \eta \omega}{2}+\frac{5 \zeta}{6}\right)+k\left(\frac{17 \delta \omega}{21}+\frac{5 i}{2}\right)-\delta \omega^{2}+2 i \eta k^{3}+\frac{5 k^{4}}{6}-\frac{5 i \omega}{2}=0 .
$$

Following for instance Charru ${ }^{25}$, the transition between convective and absolute instability is defined by the marginal mode that is neither amplified nor damped, i.e. for $\omega_{i}=0$, and located at a fixed position in space. This mode corresponds to a zero group velocity defined as $V=\partial_{k} \omega$. Considering $\omega=\omega(k)$ in (5), differentiating the equation with respect to $k$, and prescribing $\partial_{k} \omega=0$, leads to

$$
2 k\left(-\frac{\delta}{7}-\frac{9}{2} i \eta \omega+\frac{5 \zeta}{6}\right)+\frac{17}{21} \delta \omega+6 i \eta k^{2}+\frac{10 k^{3}}{3}+\frac{5 i}{2}=0 .
$$

For a given set of parameters $(\delta, \eta)$, the system of complex equations (5-6) can be solved for the four real variables $k_{r}, k_{i}, \omega_{r}$ and $\zeta$. Note that the choice of $\zeta$ as a 
Critical inclination for $\mathrm{A} / \mathrm{C}$ transition in inverted falling films

variable (instead of $\delta$ or $\eta$ ) is arbitrary but convenient. In the case of negligible viscous extensional effects, i.e. for $\eta=0$, (2) reduces to the first-order model and the system of equations to be solved for the A/C transition is merely (5-6) in which $\eta=0$. In the case of $\delta=\eta=0$ corresponding to the single long-wave equation (3), the system (5-6) reduces to

$$
\begin{aligned}
3 i \omega & =k^{4}+k^{2} \zeta+3 i k, \\
0 & =4 k^{3}+2 k \zeta+3 i .
\end{aligned}
$$

The non-trivial real solution of $(7)$, with $k_{r}>0$, is

$$
\begin{aligned}
& \zeta_{c}^{(0)}=-\frac{33^{2 / 3}}{\sqrt[3]{2(17+7 \sqrt{7})}} \approx-1.507, \quad k_{r}^{(0)}=\sqrt[6]{\frac{153}{256}+\frac{117 \sqrt{7}}{512}} \approx 1.03, \\
& k_{i}^{(0)}=-\frac{\sqrt[3]{9-3 \sqrt{7}}}{22^{2 / 3}} \approx-0.32, \quad \text { and } \quad \omega_{r}^{(0)}=\frac{1}{8} \sqrt[3]{3} \sqrt[6]{10037+3794 \sqrt{7}} \approx 0.94 .
\end{aligned}
$$

As anticipated, the solution (8) is identical to the one obtained, not only by Brun et al. for the R-T instability, but also by Duprat et al. (2007) for the Rayleigh-Plateau instability in falling films.

Solution of (5-6) that includes inertia and viscous extensional effects can now be tracked by continuation with the software AUTO-07p, using (8) as starting solution. The same procedure can also be applied to the full second-order model (1) and the system of equations to be solve in this case for the $\mathrm{A} / \mathrm{C}$ transition is given in Appendix B.

The set of parameters $(\zeta, \delta, \eta)$ used so far and referred to as the Shkadov scaling, can be converted to a set of more common parameters, namely $(C t, R e, W e)$, corresponding to the inclination number, the Reynolds number and the Weber number, respectively. The two sets of parameters are related by the following expressions :

$$
\zeta=\frac{C t}{W e^{1 / 3}}, \quad \delta=\frac{3 R e}{W e^{1 / 3}} \quad \text { and } \quad \eta=\frac{1}{W e^{2 / 3}},
$$


Critical inclination for $\mathrm{A} / \mathrm{C}$ transition in inverted falling films

whose parameters depend in turn on the physical quantities as follow :

$$
C t=\cot \beta, \quad R e=\frac{g \sin \beta h_{N}^{3}}{3 \nu^{2}} \quad \text { and } \quad W e=\frac{\gamma}{\rho g h_{N}^{2} \sin \beta},
$$

where $\nu$ is the kinematic viscosity, $\gamma$ is the surface tension and $\rho$ is the density of the

liquid. The range $0<\beta<\pi / 2$, i.e. $C t>0$, corresponds to the situation for which the hydrostatic force stabilises the flow, while the range $\pi / 2<\beta<\pi$, i.e. $C t<0$, corresponds to the situation considered in this paper for which the hydrostatic force destabilizes the flow through the R-T mechanism.

\section{RESULTS}

\section{A. Shkadov scaling}

Results for various values of $\eta$ are plotted in Figure 3. The solid lines correspond to the transition between absolute instability on the left of the curves and convective instability of the right of the curves, as calculated with the full second-order model. The dotted lines are the same transitions calculated with the simplified second-oder model. The dot-dashed curve corresponds to the first-order model, i.e. the simplified model with $\eta=0$, which slightly differs from the full second-order model with $\eta=0$ as the latter still accounts for second-order inertia effects.

Bullet points in Figure 3 indicate three particular values of $\zeta$ as follow :

$-\zeta_{\mathrm{A}} \approx-1.507$ is the solution $(8 \mathrm{~b})$ obtained in the limit of no inertia and no viscous extensional effects ;

$-\zeta_{\mathrm{B}} \approx-1.453$ is the limiting value below which the film is absolutely unstable for $\delta=0$ provided $\eta_{\mathrm{B}} \geq 0.63$;

$-\zeta_{C} \approx-1.22$ is the limiting value above which the film cannot be absolutely unstable, whatever the values of $\delta$ and $\eta$. 
Critical inclination for $\mathrm{A} / \mathrm{C}$ transition in inverted falling films
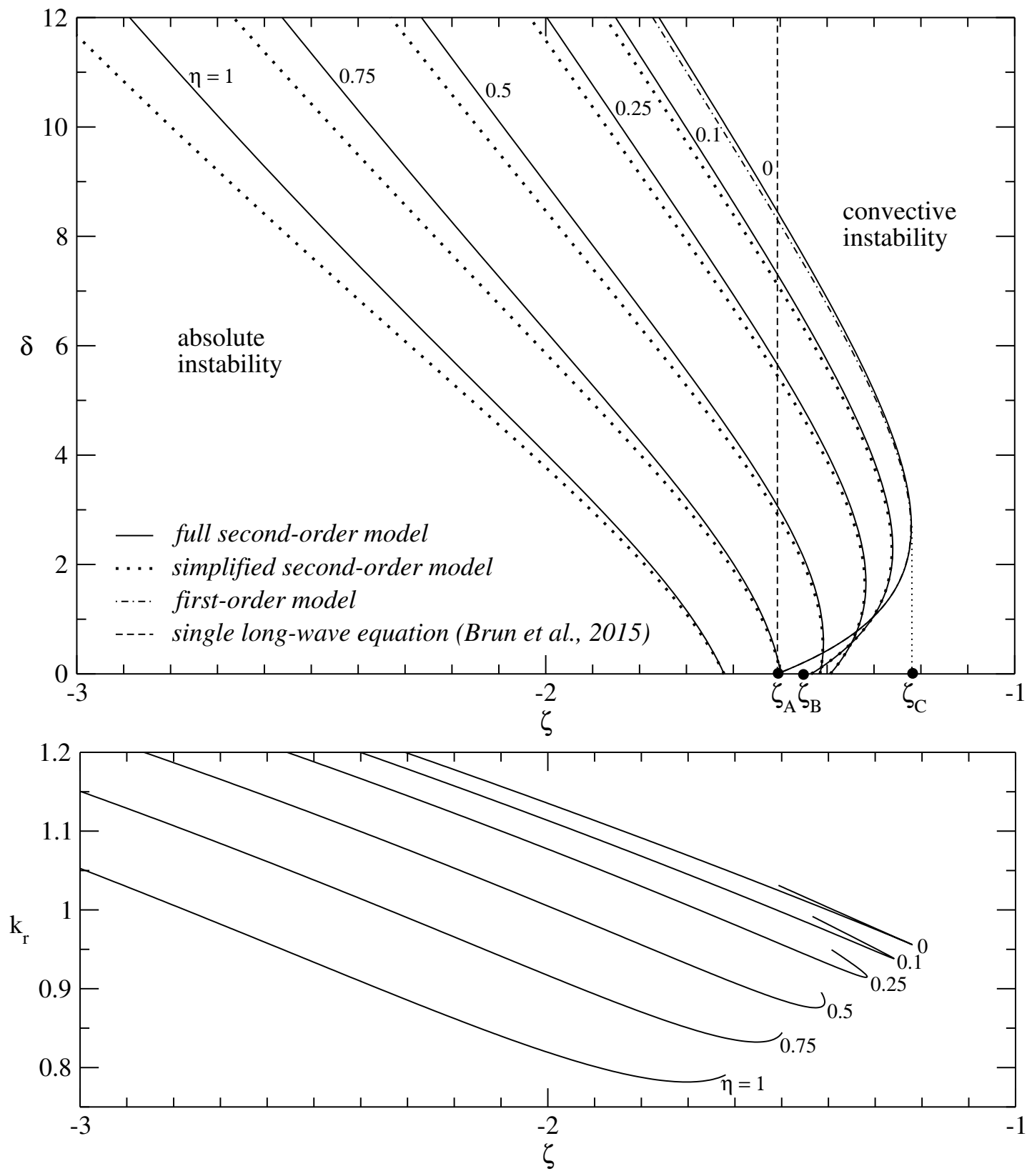

Figure 3: (top) Convective/absolute transition for various values of $\eta$ and for the different models considered in this paper. See details in text for the bullet points. (bottom) Corresponding values of the wavenumber $k_{r}$ calculated with the full second-order model. 
Critical inclination for $\mathrm{A} / \mathrm{C}$ transition in inverted falling films

The differences between the simplified and the full second-order models are negligible for $\delta<1$ but increase with $\delta$. As compared to the first-order model, viscous extensional stresses play an important role in stabilizing the flow by displacing to lower values of $\zeta$ the A/C transition. Similarly, comparing the first-order model with the single long-wave equation shows a crucial influence of inertia effect, which is nonmonotonous for $\eta<\eta_{B}=0.63$, i.e. destabilizing for low $\delta$ and stabilizing for large $\delta$, whereas it is always stabilizing for $\eta>\eta_{B}$. In other words, the turning points in some of the curves of Figure 3 indicates the presence of an absolutely unstable window in a range of $\delta$ that depends on $\zeta$ and $\eta$ but can only exist for $0<\eta<\eta_{\mathrm{B}}$ and $\zeta_{\mathrm{B}}<\zeta<\zeta_{\mathrm{C}}$.

The bottom plot in Figure 3 shows the values of the wavenumber $k_{r}$ corresponding to the A/C transition and calculated only with the full second-order model. For a practical point of view, the dimensional wavelength can be calculated as $\lambda_{r}=$ $2 \pi W e^{1 / 3} h_{N} / k_{r}$, which is in general in the millimetre range.

For an inclined plate with the liquid flowing on its underside, both the driving force entraining the liquid to flow along the plate, and the hydrostatic destabilising force are induced by gravity and antagonistically depend on the inclination angle $\beta$. Additionally, the driving force can be measured by two different ways, depending on the imposed conservation condition, namely (i) the imposed film thickness and (ii) the imposed flow rate. These conditions are separately examined in the following two subsections.

\section{B. Imposed film thickness}

The case of an imposed film thickness has recently been studied by Brun et al. ${ }^{9}$, who poured a layer of liquid on top of a glass plate and then, after a significantly long resting time, inverted the plate up to a given angle. To be consistent with their 
Critical inclination for $\mathrm{A} / \mathrm{C}$ transition in inverted falling films

study, the same set of independent parameters is adopted in this section, namely $\alpha$ for the plate inclination taken from the vertical (see Fig.1) and the initial flat film thickness parameter $h_{*}=h_{N} / \ell_{c}$, where $\ell_{c}=\sqrt{\gamma / \rho g}$ is the capillary length. Additionally, the Kapitza number is defined as $K a=\left(\ell_{c} / \ell_{\nu}\right)^{2}$, where $\ell_{\nu}=\left(\nu^{2} / g\right)^{1 / 3}$ is the viscous/gravity length, hence $K a=\gamma /\left(\rho g^{1 / 3} \nu^{4 / 3}\right)$. Using the definitions provided in (9) and (10), as well as $\beta=\alpha+\pi / 2$, yields the following relations,

$$
\zeta=-h_{*}^{2 / 3}(\cos \alpha)^{1 / 3} \tan \alpha, \quad \delta=h_{*}^{11 / 3}(\cos \alpha)^{4 / 3} K a^{3 / 2} \quad \text { and } \quad \eta=h_{*}^{4 / 3}(\cos \alpha)^{2 / 3}
$$

The limit of no inertia and no viscous extensional stress, as considered by Brun et $a l .{ }^{9}$, is recovered by rearranging the first relation in (11) and using the solution in (8) :

$$
\tan \alpha_{c}^{(0)} \sqrt{\sin \alpha_{c}^{(0)}}=\frac{\left|\zeta_{c}^{(0)}\right|^{3 / 2}}{h_{*}} \approx \frac{1.8495}{h_{*}} .
$$

To account for the effect of inertia, the value of the Kapitza number needs to be specified. Castor oil, as measured by Brun et al. ${ }^{9}$, has a capillary length of $\ell_{c}=1.91 \mathrm{~mm}$ and a viscous length of $\ell_{\nu}=4.7 \mathrm{~mm}$, with the kinematic viscosity of $0.001 \mathrm{~m}^{2} / \mathrm{s}$ at $20^{\circ} \mathrm{C}$. This yields a Kapitza number of $K a=0.17$, which is in the lower range of common values for this number.

Figure 4 shows the critical angle for the A/C instability transition versus the dimensionless thickness $h_{*}$ for various values of the Kapitza number. Obviously, and for any fixed value of $h_{*}$, the film is absolutely unstable as the plate inclination tends to the horizontal, i.e. $\alpha \rightarrow 90^{\circ}$, while there is always a critical angle $\alpha_{c}$ below which the system becomes convectively unstable. This critical angle does not vary monotonically with $h_{*}$, which constitutes one of the main finding of this work. For small values of $h_{*}$, i.e. on the left of the curve minimum, $\alpha_{c}$ decreases with increasing $h_{*}$ showing a 'destabilizing' effect of the flow, in the sense that the region of absolute instability is enlarged. For larger values of $h_{*}$, i.e. at the right of the curve minimum, 
Critical inclination for $\mathrm{A} / \mathrm{C}$ transition in inverted falling films

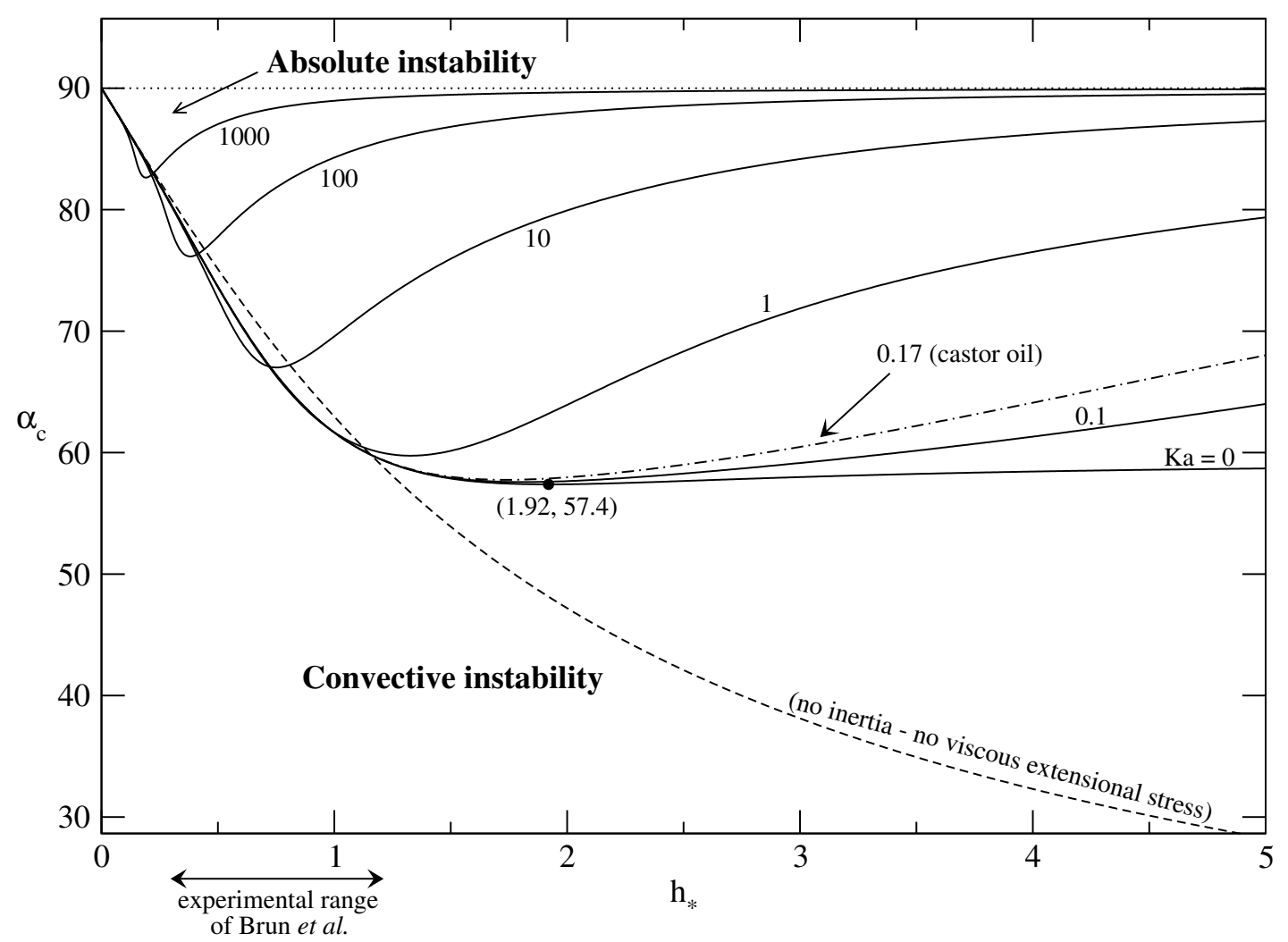

Figure 4: Critical inclination angle $\alpha_{c}$ for convective/absolute transition for various values of $K a$ as obtained with the full-second-order model (solid lines). The black dot indicates the position of the minimum in the case of $K a=0$. The curve corresponding to Castor oil is in dot-dashed line and the one corresponding to (12) obtained with the single long-wave equation, i.e. $\alpha_{c}^{(0)}$, is in dashed line.

$\alpha_{c}$ increases with $h_{*}$, which in turn shows a 'stabilizing' effect of the flow, in the sense that the region of absolute instability is reduced, even though this is less pronounced for the lowest values of the Kapitza number.

The antagonist role of the flow can be understood for instance by fixing the inclination angle and increasing $h_{*}$ from zero. Small thickness triggers small amplitude waves, which can prevent the absolute instability to occur, whereas larger thickness 
Critical inclination for $\mathrm{A} / \mathrm{C}$ transition in inverted falling films

triggers larger amplitude waves, which can reinforce the R-T instability mechanism and bring the system into the absolute instability region. As the thickness is still increased, the flow dominates the system again and brings it back into the convective instability region.

Additionally, the critical angle strongly depends on the Kapitza number. Increasing the Kapitza number decreases the region of absolute instability and sharpens the curves around their minima. These minima are also shifted to lower values of the film thickness with increasing Kapitza number, especially evident for $K a=1000$, which is in the order of magnitude of low viscous fluids and/or high surface tension, like water. Remarkably, in the limit of $K a \rightarrow 0$, this minimum critical angle does not go below an angle of $\alpha=57.3875^{\circ} \approx 57.4^{\circ}$, which is indicated by the black dot in Fig. 4. This result represents a fundamental difference with the results of Brun et $a l .{ }^{9}$ in the limit of no-inertia, which does not show any minimum (see dashed line in Fig.4). In our scaling, and as shown in (11), $K a=0$ indeed corresponds to no inertia effects since it cancels out the reduced Reynolds number $\delta$. Yet viscous extensional stress has already a strong influence in this no-inertia limit, as it prevents alone the flow to be absolutely unstable below the curve minimum (black dot).

The curve corresponding to Castor oil is represented in dot-dashed line in Fig.4, showing that the film cannot be absolutely unstable for an angle from the vertical below approximately $58^{\circ}$, which agrees with the experimental observations of Brun et $a l .{ }^{9}$. The range of thickness covered by the experiment of Brun et al. is $0.3 \leq h_{*} \leq 1.2$, in which (12) remains a fair approximation. However, and as already mentioned, for $h_{*}>1.2$ inertia and viscous extensional effects become significant, which makes the approximation (12) unusable in this range. 
Critical inclination for $\mathrm{A} / \mathrm{C}$ transition in inverted falling films

\section{Imposed flow rate}

For a film fed at a constant flow rate $q_{N}$, the relevant parameter is the Reynolds number, defined in (10) and equivalently equal to $R e=q_{N} / \nu$. Following the same procedure as in the previous section, the relations between the parameters become

$$
\zeta=-\frac{(3 R e)^{2 / 9} \sin \alpha}{K a^{1 / 3}(\cos \alpha)^{8 / 9}}, \quad \delta=\frac{(3 R e)^{11 / 9}(\cos \alpha)^{1 / 9}}{K a^{1 / 3}} \quad \text { and } \quad \eta=\frac{(3 R e)^{4 / 9}(\cos \alpha)^{2 / 9}}{K a^{2 / 3}} .
$$

The limit of no inertia and no viscous extensional stress corresponds to

$$
\left(\tan \alpha_{c}^{(0)}\right)^{4} \sqrt{\sin \alpha_{c}^{(0)}}=\left|\zeta_{c}^{(0)}\right|^{9 / 2} \frac{K a^{3 / 2}}{3 R e} \approx 2.1086 \frac{K a^{3 / 2}}{R e} .
$$

Figure 5 shows the critical angle for the A/C instability transition versus the Reynolds number $R e$ for various values of $K a$. The branches on the left of the minima for each curve (see black dots) can fairly be approximated by (14) (not shown) but again the curve minimum and the right branches are not captured by this approximation. At high Reynolds numbers, the convective transport dominates the growth of the R-T instability for which the film flow is convectively unstable. In the high Reynolds number regime, the Kapitza number (surface tension) has less significant influence, for which the curves for the various values of $K a$ more or less converge. Decreasing the flow rate in this range results also in a reduction of the propagation speed, owing to the strong correlation between wave velocity and surface velocity. As a consequence, the convective transport of the R-T instability is low. In order to remain in the regime of convective instability, the destabilizing component of gravity $g \cos \alpha$ needs to be reduced, decreasing the growth of R-T instability. Thus, the value of the critical angle reduces with the decrease of the Reynolds number down to the minimum value of the critical inclination angle. Below this value, surface tension is the main mechanism of flow stabilization as seen by the significant influence of Kapitza number. 
Critical inclination for $\mathrm{A} / \mathrm{C}$ transition in inverted falling films

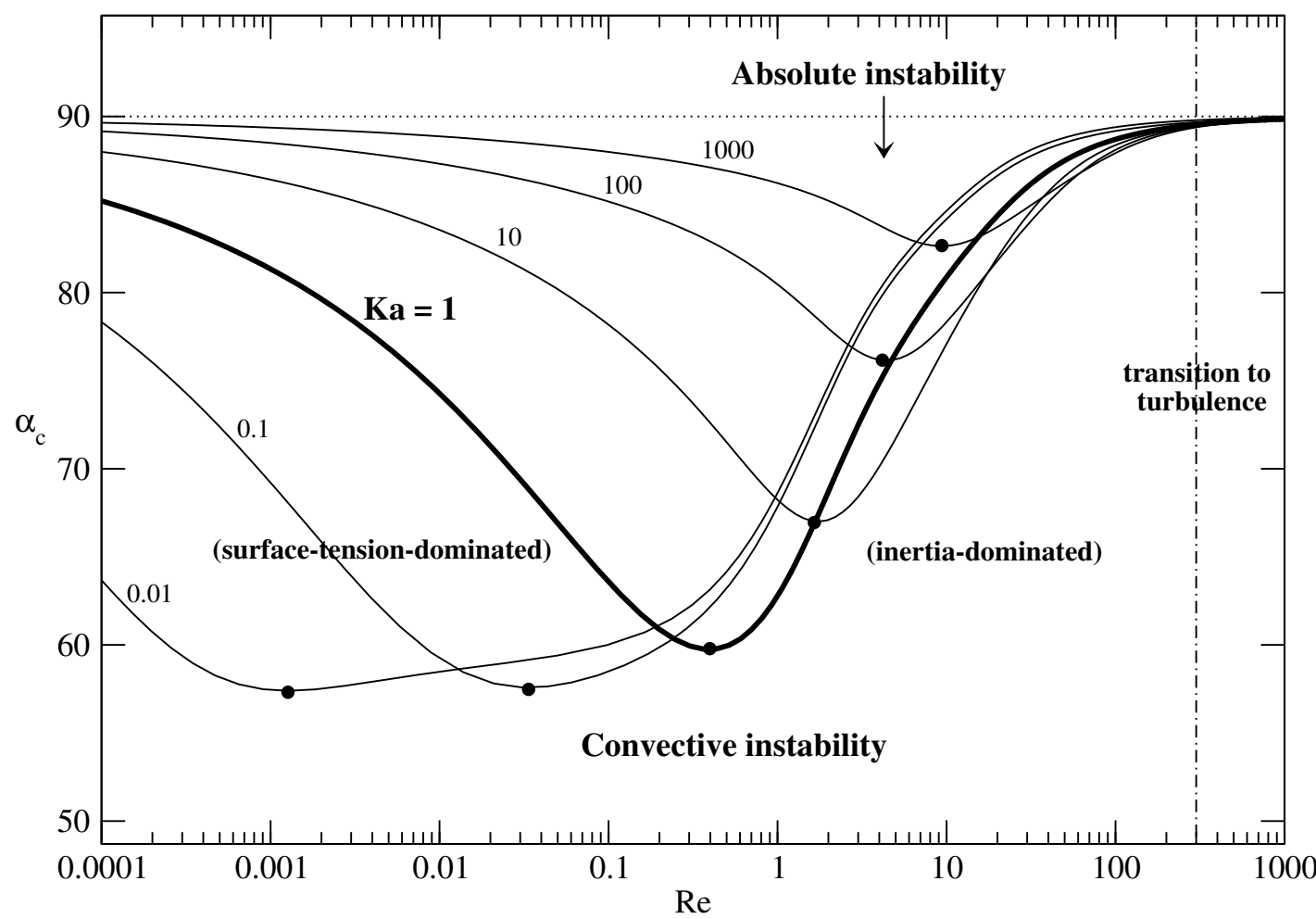

Figure 5: Convective/absolute transition for various values of $K a$ as obtained with the full-second-order model. The thick solid line for $K a=1$ is to exemplifies that the convective instability region below the curve is dominated by surface tension on the left and by inertia on the right, while the region above the curve corresponds to the absolute instability. These observations apply to all other curves. The black dots indicate the position of $\alpha_{c}^{\min }$ for each $K a$, the locus of which is continued in Fig. 6a. The vertical dot-dashed line indicates the transition for turbulence, which lies outside the range of validity of the present theory.

Based on these observations, and as exemplified in the case of $K a=1$ in Fig. 5 (thick sold line), one can state that the region of convective instability on the left of the minimum value of the critical angle is dominated by surface tension while the region of convective instability which lies on the right of this minimum is dominated 
Critical inclination for $\mathrm{A} / \mathrm{C}$ transition in inverted falling films

by inertia. The region of absolute instability in between is dominated by the negative hydrostatic forces responsible for the R-T instability.

We have drawn in Fig. 5 a vertical dot-dashed line that approximately indicates the transition between laminar and turbulent falling films ${ }^{26}$, which is approximately considered to be at $R e \approx 300$. Even though we have plotted the $\mathrm{A} / \mathrm{C}$ transition curve up to $R e=1000$ in order to show that all curves asymptotically tend to $\alpha=90^{\circ}$, these curves obviously lay outside the domain of validity of the low-dimensional models that have been used in this work. This said, and as already mentioned, comparisons of linear stability curves between the full second-order model and the OrrSommerfeld equation show excellent agreement for $R e=\mathcal{O}(100)^{3,24}$, which justifies our computations up to Reynolds numbers of the same order of magnitude.

\section{Minimum critical angle}

In the previous subsections, Figs. 4 and 5 showed that for each value of the Kapitza

number a minimum critical angle $\alpha_{c}^{\min }$ is given below which the falling film is always convectively unstable, independently of the conservation condition (constant flow rate or constant film thickness). Figure 6a shows the locus of this minimum critical angle in the entire range of Kapitza numbers. Now, above the minimum critical angle, the film can either be convectively or absolutely unstable, depending on the flow parameters, i.e. $h_{*}$ or Re. What we have learned from Figs. 5 is that there is a region of absolute instability that separates a region of convective instability dominated by surface tension and a region of convective instability dominated by inertia. Plotting in Fig. $6 \mathrm{~b}$ and $\mathrm{c}$ the position $h_{*}^{\min }$ and $R e^{\mathrm{min}}$, respectively, corresponding to the minimum critical angle allows to discriminate in the parameter space between the two regions.

In Fig. 6a, the minimum critical angles are indicated by black dots for castor oil 
Critical inclination for $\mathrm{A} / \mathrm{C}$ transition in inverted falling films

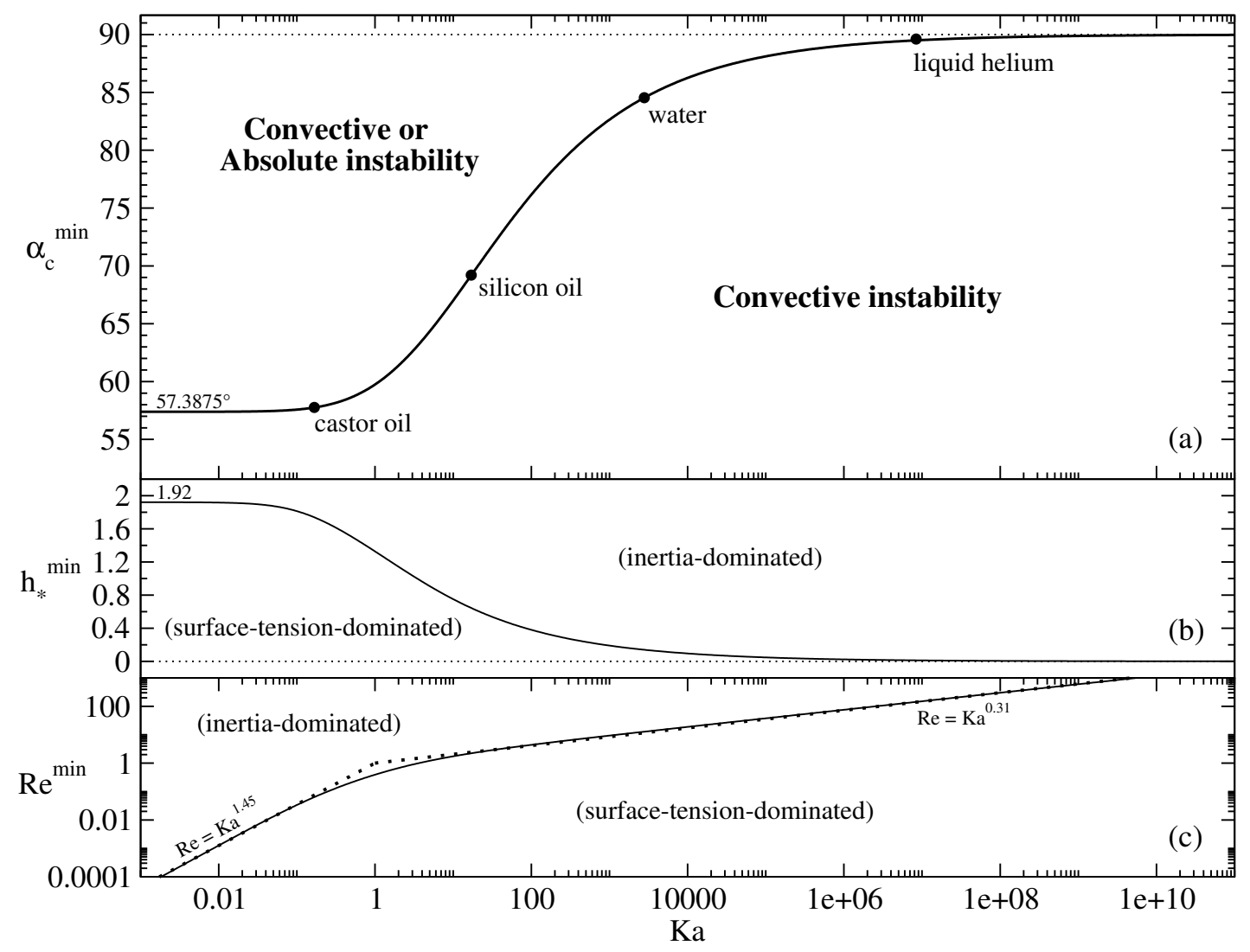

Figure 6: Minimum critical angle between absolute and convective instability, in function of the Kapitza number (a), along which the corresponding values of the dimensionless film thickness (b) or alternatively the Reynolds number (c). In (c), thick dotted lines are fitted power trends together with their expressions.

$(K a=0.17)$, silicon oil $(K a=17.8)$, water at $20^{\circ} C(K a=3923)$, and liquid helium at $5 \mathrm{~K}\left(\mathrm{Ka}=8.6 \times 10^{6}\right)$. As already pointed out, the most salient feature is that there is an angle $\left.\alpha_{\mathrm{c}}^{\min }\right|_{K a \rightarrow 0}=57.3875^{\circ}$ obtained in the limit of $K a \rightarrow 0$ corresponding to an infinitely viscous fluid below which none of the liquid film systems can be absolutely unstable. For real liquids of finite viscosity, such as for silicon oil, this minimum critical angle is $69^{\circ}$, for water it is $84.5^{\circ}$ and for liquid helium it is $89^{\circ}$. 
Critical inclination for $\mathrm{A} / \mathrm{C}$ transition in inverted falling films

\section{DISCUSSION}

Our approach for computing the A/C instability transition curves has implicitly consisted in tracking by continuation the dominant saddle point in the complex plane $\left(k_{r}, k_{i}\right)$, which is the one having the highest growth rate $^{27}$, namely the one

satisfying the so-called "collision criterion" established by Briggs ${ }^{28}$. The starting saddle point for the tracking method was the one obtained analytically in the limit of no inertia $(\delta \rightarrow 0)$ and no extensional stress $(\eta \rightarrow 0)$ and given by (8). The symmetry properties of the dispersion relation for the single long-wave equation (7a) ensures the obtained saddle-point to be the only viable candidate. This is no longer true for the dispersion relations of the simplified and the full second-order models, i.e. (5) and (B1a), respectively. There are other saddle points and we have therefore no guarantee that the one found by continuation is effectively the dominant one in the entire parameter domain explored in this study. Some examples in the literature indeed show that misleading or subdominant saddle points had to be disregarded ${ }^{29-32}$.

Instead of looking at the structure of the spatial branches throughout the entire continuation procedure, which represents a tedious task, we propose here another method to verify that the $\mathrm{A} / \mathrm{C}$ transition identified in this paper corresponds to the dominant saddle point. For that purpose, we compute the system impulse response using direct numerical simulation. More specifically, we employ Gerris which is an open-source software that uses the VOF method and adaptive refinement of quadtree meshes $^{33}$. The initial state of our computations is a uniform film in a long periodic domain with a small amplitude sharp gaussian pulse situated at one forth of the domain length. We adjust the pulse amplitude for each simulation in order to visualize the evolution of the wave packet. Even though we cannot distinguish with this approach between the linear and the nonlinear impulse response of the flow, these properties coincide with each other most of the time ${ }^{34}$, as it is assumed to be the 
Critical inclination for $\mathrm{A} / \mathrm{C}$ transition in inverted falling films

case here.

Figure $7(\mathrm{a})$ compares for a fixed Kapitza number the transition curve obtained with the full second-order model to the impulse response behaviour of the flow computed with Gerris. The agreement is convincing especially in the sense that it captures well the minimum of the $\mathrm{A} / \mathrm{C}$ transition curve, which is the main difference between our theory and the one by Brun et $a .^{9}{ }^{9}$. Very close to the curve, it becomes difficult to conclude on the absolute/convective nature of the response because the edges of the wavepacket oscillate (see figures $7 \mathrm{~b}$ and c). These oscillations are simply due to the way the edges (dashed lines) are constructed, based on the following threshold criterion : $|h-1|=A$, with $5 \times 10^{-3}<A<5 \times 10^{-2}$ adjusted for the different inclination angles and Reynolds numbers. Notice that the analysis has been restricted to the range $1<R e<60$. For $R e<1$, the instability growth rate is very low which means long computational time. On the contrary for $R e>60$, the instability growth rate is very large and numerical noise is rapidly amplified. Note that for $R e<1$, the transition curve obtained with the full second-order model is very close to the one obtained with the single long-wave equation ${ }^{9}$, which is a proof by itself that the dominant saddle-point is well captured in this range.

\section{CONCLUSIONS}

This study examined the critical inclination angle for the transition from absolute to convective instability of falling liquid films flowing on the underside of a plate. The results are based on the weighted integral boundary layer models which has been proven by many studies to give reliable results in the laminar regime of falling liquid

films. The transition between the absolute and convective instability is obtained by tracking the marginal mode that is neither amplified nor damped and located at a fixed position in space. Tracking the transition for the case of a constant film thi- 
Critical inclination for $\mathrm{A} / \mathrm{C}$ transition in inverted falling films

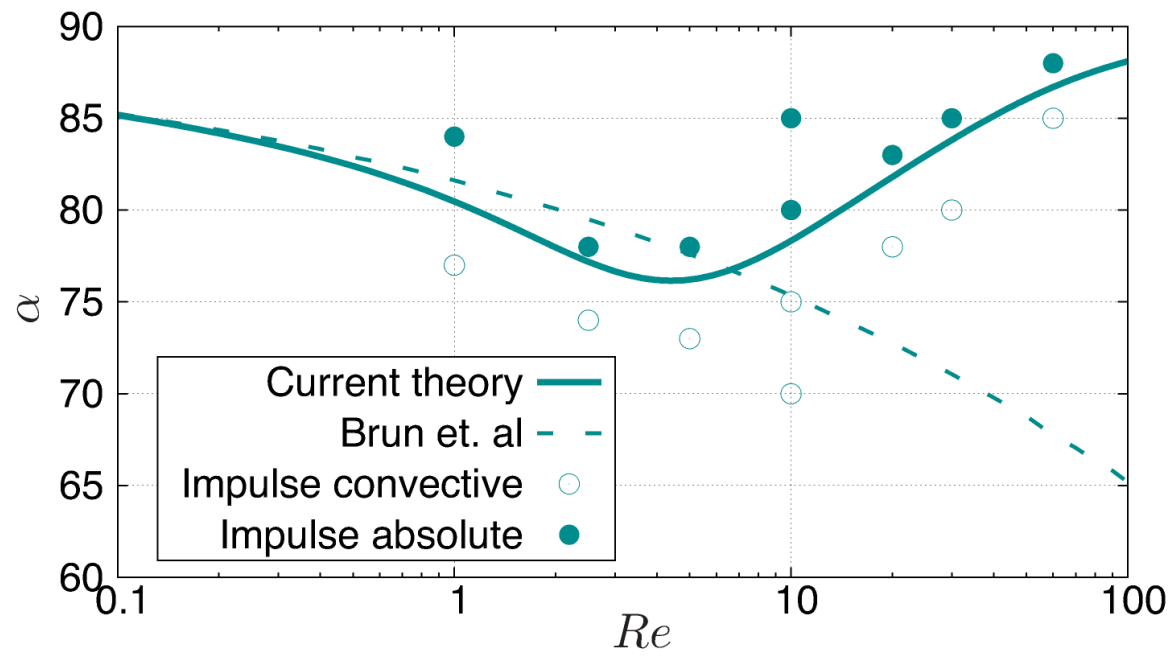

(a)

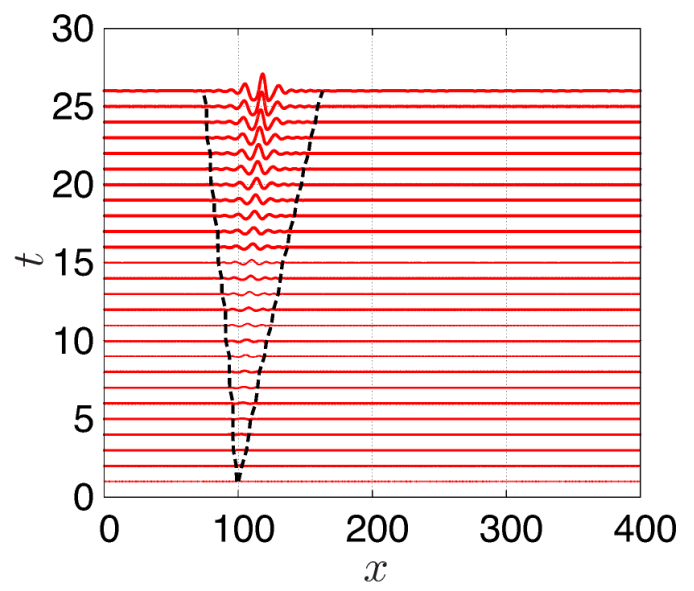

(b)

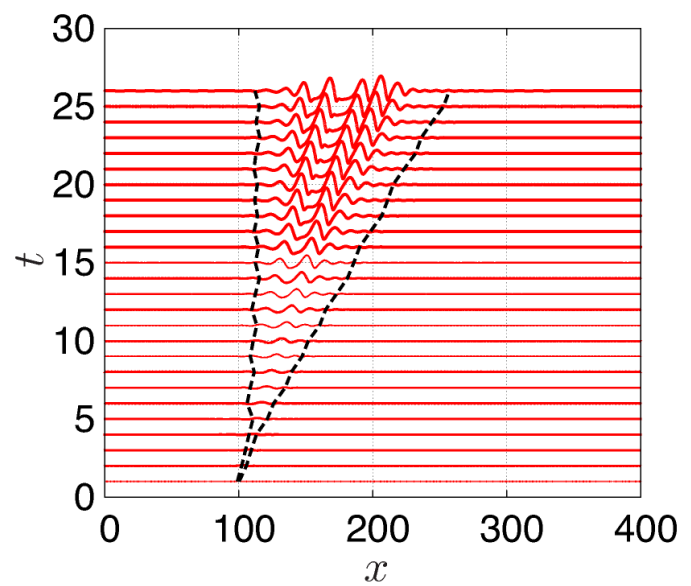

(c)

FiguRE 7: Verification of the current theory using direct numerical simulations of the impulse response computed with Gerris for $K a=100$ : (a) comparison to the transition curve obtained with the full second-order model, (b) spatio-temporal diagram showing an absolute instability behaviour for $\alpha=85^{\circ}, R e=10$, and (c) a convective instability behaviour for $\alpha=70^{\circ}, R e=10$. The dashed lines in (b) and (c) show the edges of the wavepacket. 
Critical inclination for $\mathrm{A} / \mathrm{C}$ transition in inverted falling films

ckness, a strong dependency on Kapitza number has been obtained. Furthermore, a significant effect of viscous extensional stress has been identified, expecially for a dimensionless film thickness larger than unity. If the flow rate is imposed as a conservation condition, the stabilizing mechanism of surface tension (dependent on Kapitza number) becomes apparent for low values of the Reynolds number. Contrarily, for high Reynolds number, the convective transport (hence inertia effects) dominates such that surface tension is of minor importance.

The A/C transition has been found by continuation of the most dominant saddle point from the limit of no inertia and no viscous extensional stress, i.e. by continuously increasing $\delta$ and $\eta$ from zero. The validity of this approach has been checked by computing the system impulse response using direct numerical simulation. The agreement for the $\mathrm{A} / \mathrm{C}$ transition is convincing over the entire range of relevant Reynolds numbers.

Finally, a minimum critical angle depending on the Kapitza number has been identified which is independent from the conservation condition (imposed flow rate or imposed film thickness). Since the Kapitza number depends on the fluid properties and on gravity only, each fluid has its own minimum critical angle on earth. This minimum critical angle decreases with Kapitza number, such that a higher gravitational force driving the flow is necessary for low Kapitza number fluids. Contrarily, high Kapitza number fluids such as Water $\left(\mathrm{Ka}=3923\right.$ at $\left.20^{\circ} \mathrm{C}\right)$, where surface tension stabilizes the flow, allow for high inclination angles of approximately $85^{\circ}$ before the instability changes from convective to absolute. This might have a practical interest in architectural applications as a condensed film forming on a ceiling would never be absolutely unstable if the ceiling is inclined by an angle of about $5^{\circ}$ from the horizontal. This is the reason why the ceiling of hammams are always slightly inclined. The determination of the critical angle in falling films evaporators that often have some section with negative hydrostatic forces could help in enhancing the design of 
Critical inclination for $\mathrm{A} / \mathrm{C}$ transition in inverted falling films

these devices and avoid clogging.

\section{ACKNOWLEDGMENTS}

We are deeply grateful to François Gallaire for fruitful discussions. We acknowledge the financial support of EU-FP7 ITN Multiflow as well as the BELSPO agency under the grant IAP-7/38 MicroMAST. B.S. also thanks the F.R.S.-FNRS for financial support. This research has been performed under the umbrella of the COST action MP1106.

\section{Annexe A: Full second-order model}

Following the weighted residuals methodology detailed e.g. in Kalliadasis et al. ${ }^{3}$, and using the two-dimensional system of reference as defined in Fig. 1(a), the streamwise velocity field is projected onto the following polynomials :

$$
\begin{aligned}
& F_{0}=\bar{y}-\frac{1}{2} \bar{y}^{2} \\
& F_{1}=\bar{y}-\frac{17}{6} \bar{y}^{2}+\frac{7}{3} \bar{y}^{3}-\frac{7}{12} \bar{y}^{4} \\
& F_{2}=\bar{y}-\frac{13}{2} \bar{y}^{2}+\frac{57}{4} \bar{y}^{3}-\frac{111}{8} \bar{y}^{4}+\frac{99}{16} \bar{y}^{5}-\frac{33}{32} \bar{y}^{6} .
\end{aligned}
$$

The streamwise velocity distribution thus reads

$$
u=\frac{3}{h}\left(q-s_{1}-s_{2}\right) g_{0}(\bar{y})+45 \frac{s_{1}}{h} g_{1}(\bar{y})+210 \frac{s_{2}}{h} g_{2}(\bar{y})
$$

where $\bar{y}=y / h$ and the flow rate $q(x, t)=\int_{0}^{h(x)} u(x, y, t) \mathrm{d} y$ appears with two corrections, namely $s_{1}$ and $s_{2}$.

Applying the Galerkin method, which consists of integrating the classical boundarylayer equations across the film, substituting the projections (A2) into the integrated equations, taking the test functions (A1) as weight functions, and using the no-slip 
Critical inclination for $\mathrm{A} / \mathrm{C}$ transition in inverted falling films

boundary condition at the wall and the stress-free condition at the interface yield the full second-order model given in (1). Note that (1b) is different than the equation for $q$ given in Ruyer-Quil and Manneville ${ }^{21}$ because the terms $\delta \partial_{t} s_{1}$ and $\delta \partial_{t} s_{2}$ have not been substituted here by (1c) and (1d), respectively, in order to enable the straightforward adiabatic elimination of $s_{1}$ and $s_{2}$ leading to the simplified second-order model.

\section{Annexe B: System of equations for the A/C transition with the full} second-order model

$$
\begin{aligned}
& 0=3 i \omega+\frac{18 \delta \omega^{2}}{13}-\frac{10}{143} i \delta^{2} \omega^{3}-\frac{4 \delta^{3} \omega^{4}}{6435}+k\left(\frac{4 \delta^{3} \omega^{3}}{5005}+\frac{98 i \delta^{2} \omega^{2}}{1287}-\frac{174 \delta \omega}{143}-3 i\right) \\
& +k^{2}\left(-\frac{2027 i \delta^{2} \eta \omega^{3}}{720720}+\omega\left(-\frac{2936 i \delta^{2}}{117117}+\frac{4 i \delta \zeta}{65}+\frac{27 i \eta}{5}\right)\right. \\
& \left.+\omega^{2}\left(-\frac{68 \delta^{3}}{195195}+\frac{3 \delta^{2} \zeta}{5005}+\frac{1077 \delta \eta}{3640}\right)+\frac{166 \delta}{715}-\zeta\right) \\
& +k^{3}\left(\frac{3439 i \delta^{2} \eta \omega^{2}}{1310400}+\frac{152 i \delta^{2}}{65065}+\omega\left(\frac{368 \delta^{3}}{6441435}-\frac{16 \delta^{2} \zeta}{45045}-\frac{2441 \delta \eta}{12012}\right)-\frac{304 i \delta \zeta}{15015}-\frac{12 i \eta}{5}\right) \\
& +k^{4}\left(-\frac{16 \delta^{3}}{6441435}+\omega\left(\frac{4 i \delta}{65}-\frac{4591 i \delta^{2} \eta}{5855850}\right)+\frac{3 \delta^{2} \omega^{2}}{5005}+\frac{148 \delta^{2} \zeta}{2927925}+\frac{10331 \delta \eta}{320320}-1\right) \\
& +k^{5}\left(\frac{197 i \delta^{2} \eta}{2602600}-\frac{16 \delta^{2} \omega}{45045}-\frac{304 i \delta}{15015}\right)+k^{6} \frac{148 \delta^{2}}{2927925}, \\
& 0=-3 i-\frac{174 \delta \omega}{143}+\frac{98 i \delta^{2} \omega^{2}}{1287}+\frac{4 \delta^{3} \omega^{3}}{5005}+k\left(-\frac{2027 i \delta^{2} \eta \omega^{3}}{360360}+2 \omega\left(-\frac{2936 i \delta^{2}}{117117}+\frac{4 i \delta \zeta}{65}+\frac{27 i \eta}{5}\right)\right. \\
& \left.+2 \omega^{2}\left(-\frac{68 \delta^{3}}{195195}+\frac{3 \delta^{2} \zeta}{5005}+\frac{1077 \delta \eta}{3640}\right)+\frac{332 \delta}{715}-2 \zeta\right) \\
& +k^{2}\left(\frac{3439 i \delta^{2} \eta \omega^{2}}{436800}+\frac{456 i \delta^{2}}{65065}+3 \omega\left(\frac{368 \delta^{3}}{6441435}-\frac{16 \delta^{2} \zeta}{45045}-\frac{2441 \delta \eta}{12012}\right)-\frac{304 i \delta \zeta}{5005}-\frac{36 i \eta}{5}\right) \\
& +k^{3}\left(-\frac{64 \delta^{3}}{6441435}+4 \omega\left(\frac{4 i \delta}{65}-\frac{4591 i \delta^{2} \eta}{5855850}\right)+\frac{12 \delta^{2} \omega^{2}}{5005}+\frac{592 \delta^{2} \zeta}{2927925}+\frac{10331 \delta \eta}{80080}-4\right)
\end{aligned}
$$


Critical inclination for $\mathrm{A} / \mathrm{C}$ transition in inverted falling films

$$
+k^{4}\left(\frac{197 i \delta^{2} \eta}{520520}-\frac{16 \delta^{2} \omega}{9009}-\frac{304 i \delta}{3003}\right)+k^{5} \frac{296 \delta^{2}}{975975}
$$

\section{RÉFÉRENCES}

${ }^{1}$ H.-C. Chang and E. Demekhin, Complex Wave Dynamics on Thin Films (D. Möbius and R. Miller, Elsevier, Amsterdam, 2002).

${ }^{2}$ S. V. Alekseenko, V. E. Nakoryakov, and B. G. Pokusaev, Wave flow of liquid films, edited by T. Fukano (Begell House, New York, 1994).

${ }^{3}$ S. Kalliadasis, C. Ruyer-Quil, B. Scheid, and M. Velarde, Falling liquid films (Spinger-Verlag, 2012) p. 440.

${ }^{4}$ G. F. Dietze, A. Leefken, and R. Kneer, "Investigation of the backflow phenomenon in falling liquid films," Journal of Fluid Mechanics 595, 435 - 459 (2008).

${ }^{5} \mathrm{~W}$. Rohlfs and B. Scheid, "Phase diagram for the onset of circulating waves and flow reversal in inclined falling films," Journal of Fluid Mechanics 763, 322-351 (2015).

${ }^{6}$ J. Liu, J. D. Paul, and J. P. Gollub, "Measurements of primary instability of film flows," Journal of Fluid Mechanics 250, 69-101 (1993).

${ }^{7}$ L. Rayleigh, "Investigation of the character of the equilibrium of an incompressible heavy fluid of variable density," Proceedings of the London mathematical society 14, 170-177 (1883).

${ }^{8} \mathrm{~S}$. Chandrasekhar, Hydrodynamics and hydromagnetic stability (Oxford University Press, 1961).

${ }^{9}$ P.-T. Brun, A. Damiano, P. Rieu, G. Balestra, and F. Gallaire, "Rayleigh-Taylor instability under an inclined plane," Physics of Fluids (2015).

${ }^{10}$ A. L. Frenkel and D. Halpern, "On saturation of rayleigh-taylor instability," in IUTAM Symposium on Nonlinear Waves in Multi-Phase Flow, Fluid Mechanics 
Critical inclination for $\mathrm{A} / \mathrm{C}$ transition in inverted falling films

and Its Applications, Vol. 57, edited by H.-C. Chang (Springer Netherlands, 2000) pp. 69-79.

${ }^{11}$ M. Fermigier, L. Limat, J. E. Wesfreid, P. Boudinet, and C. Quilliet, "Twodimensional patterns in rayleigh-taylor instability of a thin layer," Journal of Fluid Mechanics 236, 349-383 (1992).

${ }^{12}$ G. H. Wolf, "Dynamic stabilization of interchange instability of a liquid-gas interface," Physics Review Letter 24, 444-446 (1970).

${ }^{13}$ E. Talib and A. Juel, "Instability of a viscous interface under horizontal oscillation," Physics of Fluids 19, 092102 (2007).

${ }^{14}$ N. A. Bezdenezhnykh, V. A. Briskman, A. A. Cherepanov, and M. T. Sharov, "Control of the stability of liquid surfaces by means of variable fields," Fluid Mech. Sov. Res. 15, 11-32 (1986).

${ }^{15}$ B. K. Kopbosynov and V. V. Pukhnachev, "Thermocapillary flow in thin liquid films," Fluid Mech. Sov. Res. 95 (1986).

${ }^{16}$ R. J. Deissler and A. Oron, "Stable localized patterns in thin liquid films," Physical Review Letters 68, 2948-2951 (1992).

${ }^{17}$ A. J. Babchin, A. L. Frenkel, B. G. Levich, and G. I. Sivashinsky, "Nonlinear saturation of Rayleigh-Taylor instability in thin films," Physics of Fluids 26, 31593161 (1983).

${ }^{18}$ T.-S. Lin, L. Kondic, and A. Filippov, "Thin films flowing down inverted substrates : Three-dimensional flow," Physics of Fluids 24, 022105 (2012).

${ }^{19}$ S. J. Leib and M. E. Goldstein, "Convective and absolute instability of a viscous liquid jet," Physics of Fluids 29, 952-954 (1986).

${ }^{20}$ J. Eggers and E. Villermaux, "Physics of liquid jets," Reports on Progress in Physics 71, 036601 (2008).

${ }^{21}$ C. Ruyer-Quil and P. Manneville, "Improved modeling of flows down inclined planes," The European Physical Journal B - Condensed Matter and Complex Sys- 
Critical inclination for $\mathrm{A} / \mathrm{C}$ transition in inverted falling films

tems 15, 357-369 (2000).

${ }^{22}$ P. Howell, "Models for thin viscous sheets," Euro. J. Appl. Math. 7, 321-343 (1996).

${ }^{23}$ C. Duprat, C. Ruyer-Quil, S. Kalliadasis, and F. Giorgiutti-Dauphiné, "Absolute and Convective Instabilities of a Viscous Film Flowing Down a Vertical Fiber," Phys. Rev. Lett. 98, 244502 (2007).

${ }^{24}$ C. Ruyer-Quil and P. Manneville, "Further accuracy and convergence results on the modeling of flows down inclined planes by weighted-residual approximations," Physics of Fluids (1994-present) 14, 170-183 (2002).

${ }^{25}$ F. Charru, Hydrodynamics Instability (Cambridge University Press, 2011).

${ }^{26}$ H.-C. Chang, "Wave evolution on a falling film," Ann. Rev. Fluid Mech. 26, 103136 (1994).

${ }^{27}$ P. Huerre and P. A. Monkewitz, "Local and global instabilities in spatially developing flows," Annu. Rev. Fluid Mech. 22, 473-537 (1990).

${ }^{28}$ R. Briggs, Electron-Stream Interaction with Plasmas (MIT Press, Cambridge, 1964).

${ }^{29}$ J. J. Healey, "On the relation between the viscous and inviscid absolute instabilities of the rotating-disk boundary layer," J. Fluid Mech. 511, 179-199 (2004).

${ }^{30} \mathrm{~J}$. Healey, "Destabilizing effects of confinement on homogeneous mixing layers," J. Fluid Mech. 623 (2009).

${ }^{31}$ M. Juniper, "The effect of confinement on the stability of two-dimensional shear flows," J. Fluid Mech. 565 (2006).

${ }^{32}$ C. Leclercq, B. Pier, and J. Scott, "Absolute instabilities in eccentric TaylorCouette-Poiseuille flow," J. Fluid Mech. 741, 543-566 (2014).

${ }^{33} \mathrm{~S}$. Popinet, "An accurate adaptive solver for surface-tension-driven interfacial flows," J. Comput. Phys. 228, 5838-5866 (2009).

${ }^{34}$ I. Delbende and J. M. Chomaz, "Nonlinear convective/absolute instabilities in parallel two-dimensional wakes," Phys. Fluids 10, 2724 (1998). 